\title{
Cornering metastases: therapeutic targeting of circulating tumor cells and stem cells
}

\section{Bishoy Faltas*}

Division of Hematology and Medical Oncology, Weill Cornell Medical College, New York, NY, USA

\section{Edited by:}

Michael R. King, Cornell University, USA

\section{Reviewed by:}

Michael R. King, Cornell University, USA

Jiahe Li, Cornell University, USA

\section{*Correspondence:}

Bishoy Faltas, Division of Hematology and Medical Oncology, Weill Cornell Medical College, New York, NY 10065 USA.e-mail: beshoyso@yahoo.com
The last decade has witnessed an evolution of our understanding of the biology of the metastatic cascade. Recent insights into the metastatic process show that it is complex, dynamic, and multi-directional. This process starts at a very early stage in the natural history of solid tumor growth leading to early development of metastases that grow in parallel with the primary tumor. The role of stem cells in perpetuating cancer metastases is increasingly becoming more evident. At the same time, there is a growing recognition of the crucial role circulating tumor cells (CTCs) play in the development of metastases. These insights have laid the biological foundations for therapeutic targeting of CTCs, a promising area of research that aims to reduce cancer morbidity and mortality by preventing the development of metastases at a very early stage. The hematogenous transport phase of the metastatic cascade provides critical access to CTCs for therapeutic targeting aiming to interrupt the metastatic process. Recent advances in the fields of nanotechnology and microfluidics have led to the development of several devices for in vivo targeting of CTC during transit in the circulation. Selectin-coated tubes that target cell adhesion molecules, immuno-magnetic separators, and in vivo photo-acoustic flow cytometers are currently being developed for this purpose. On the pharmacological front, several pharmacological and immunological agents targeting cancer stem cells are currently being developed. Such agents may ultimately prove to be effective against circulating tumor stem cells (CTSCs). Although still in its infancy, therapeutic targeting of CTCs and CTSCs offers an unprecedented opportunity to prevent the development of metastasis and potentially alter the natural history of cancer. By rendering cancer a "local" disease, these approaches could lead to major reductions in metastasis-related morbidity and mortality.

Keywords: circulating tumor cells, cancer stem cell, epithelial-mesenchymal transition, immuno-magnetic separation, selectin ligands, targeted therapy, metastasis

\section{THE BIOLOGY OF THE METASTATIC CASCADE}

The metastatic spread of cancer is the main cause of morbidity and mortality in cancer patients (Duffy et al., 2008). The development of metastases from solid tumors is a complex multi-step process and is one of the most enigmatic aspects of cancer biology. Recently, studies using in vivo vital microscopy have shed more light on the physiology of the metastasis. These studies show a metastatic process that proceeds in a pre-determined cascade comprising the following steps: neoangiogenesis, trans-endothelial migration, entry into the blood stream (intravasation), transport through the vasculature followed by extravasation, and ultimately resulting in colonization and growth at distant sites (Chambers et al., 1995; Morris et al., 1997; Figure 1).

Although the biology of the metastatic process is not fully understood, new insights are emerging from recently discovered metastatic phenomena. For instance, it was previously believed that the development of metastases is a late event that occurs only after primary tumors reach a certain critical mass (the linear-progression model). Recent evidence indicates that tumor cells disseminate at a relatively early stage of the natural history of tumor growth. This leads to parallel growth of metastasis along with primary tumor growth (the parallel-progression model; Klein, 2009). Similarly, metastatic spread has been classically viewed as a unidirectional process with dissemination of cancer cells occurring only from primary tumors to form secondary metastases. On the contrary, recent experimental evidence points toward a new paradigm where cancer progress is a multidirectional process in which tumor cells are capable of self-seeding the primary tumor (Kim et al., 2009; Comen et al., 2011).

The metastatic process is selective and inefficient with many cancer cells initially gaining access to the circulation but only a few ultimately succeeding in proliferating as distant metastases (Wong et al., 2001). In fact, it is estimated that only $1 \%$ of micrometastases eventually thrive as macro-metastases (Wittekind and Neid, 2005). Despite this relative metastatic inefficiency, the absolute magnitude of metastatic spread is enormous and represents the primary cause of cancer morbidity and mortality (Wittekind and Neid, 2005; Chaffer and Weinberg, 2011).

\section{THERAPEUTIC TARGETING OF THE METASTATIC CASCADE}

Despite the tremendous toll of metastases on human mortality and morbidity, the development of preventive anti-metastatic therapies has been slow and often hampered by the lack of understanding of the biology of the metastatic cascade (Mina and 


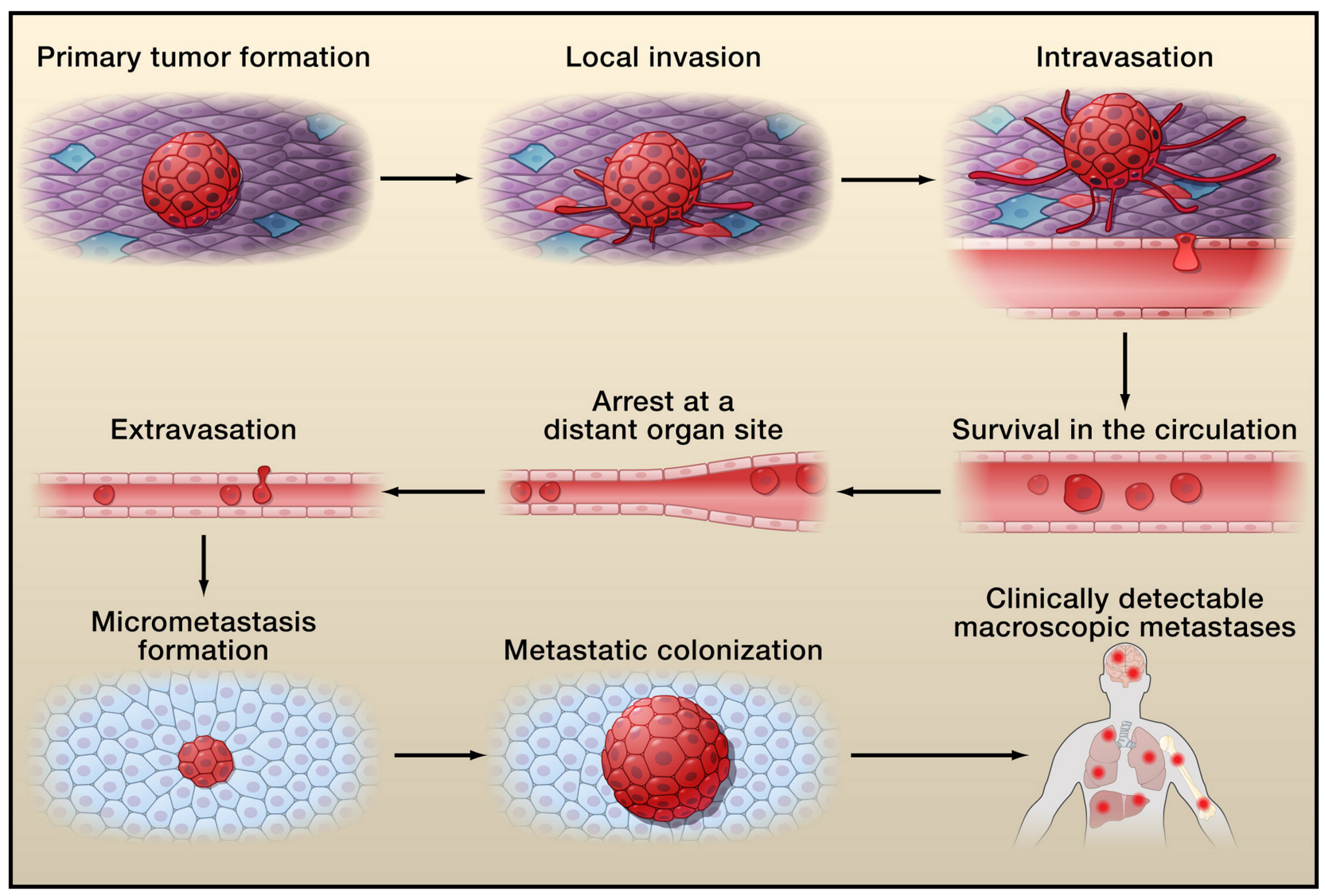

FIGURE 1 | The invasion-metastasis cascade. Tumor cells exit their primary sites of growth (local invasion, intravasation), translocate systemically (survival in the circulation, arrest at a distant organ site, extravasation), and adapt to survive in distant tissues (micro-metastasis formation, metastatic colonization). Adapted with permission from Valastyan and Weinberg (2011).
Sledge, 2011). Most currently developed anti-metastatic therapy strategies target the final steps of the metastatic cascade such as extravasated dormant cancer cells in the metastatic niche thus missing the opportunity to interrupt metastases development at earlier stages (Chambers et al., 2000; MacDonald et al., 2002). Targeting earlier "up-stream" events in the metastatic cascade is likely to be more effective in preventing metastases from developing with greater yield in reducing or even eliminating their devastating clinical impact. The hematogenous transport phase of cancer cells presents a particularly attractive opportunity for therapeutic targeting for multiple reasons; first, it occurs early enough to provide a rational up-stream target for strategies aiming to reduce or completely prevent the development of distant metastases. Secondly, hematogenous transport represents the final common pathway of metastatic spread, even for tumors initially spreading through lymphatics which eventually inter-connect with the circulatory system (Sleeman et al., 2011). Finally, the transport phase is relatively accessible to a variety of pharmacological and mechanical targeting interventions.

\section{CIRCULATING TUMOR CELLS AS THERAPEUTIC TARGETS}

Circulating tumor cells (CTCs) are cancer cells that detach from the primary tumor and enter the bloodstream. Several systems have been developed for isolation and characterization of CTCs from blood samples including the FDA approved CellSearch ${ }^{\circledR}$ platform (Hughes and King, 2012; Yu et al., 2011). Using these platforms, CTCs have been isolated from blood samples of patients with a variety of tumors including head and neck (Nichols et al., 2011), breast (Swaby and Cristofanilli, 2011), lung (O'Flaherty et al., 2011), colorectal, gastric, pancreatic (Takeuchi and Kitagawa, 2010), renal cell, urinary bladder, and prostate cancers (Kruck et al., 2011). Recent studies show significant correlation between higher CTC counts and shorter overall survival in patients with metastatic breast, lung, and prostate carcinomas (Cristofanilli et al., 2004; Miller et al., 2010). These studies support a critical role for CTCs in tumor progression and metastases. As a result, it is now hypothesized that targeting CTCs during their hematogenous transport could lead to effective interruption of the metastatic cascade and ultimately, reduction of cancer morbidity and mortality (Faltas, 2010).

\section{CIRCULATING TUMOR STEM CELLS AND EPITHELIAL-MESENCHYMAL TRANSITION}

Circulating tumor cells occur in heterogeneous populations with pronounced differences in their metastatic potential. Some CTC populations undergo a transformative process called 
epithelial-mesenchymal transition (EMT) that leads to acquisition of enhanced migratory and invasive properties (Bonnomet et al., 2010). Cells undergoing EMT also acquire cancer stem cell (CSC)-like characteristics including the capacity for selfrenewal, re-differentiation, dormancy, active DNA repair, drug resistance, and resistance to apoptosis leading to resistance to chemotherapeutic agents (Monteiro and Fodde, 2010; Chaffer and Weinberg, 2011). These CTCs possessing stem cell like characteristics will be referred to in this review as circulating tumor stem cells (CTSCs). Because of these unique characteristics, CTSCs are considered high-value targets for anti-metastatic therapies. Unfortunately, the biology of this unique subset of cells is not fully understood, posing a challenge to precise identification of cell populations most likely to initiate metastases in distant organs or those that are most difficult to eliminate using existing chemotherapeutic approaches (Faltas et al., 2011). Several markers have been proposed to identify CTSCs including CD133, a glycoprotein expressed on hematopoietic progenitors. In one study, real-time reverse-transcriptase polymerase chain reaction was used to detect mRNA of CD133 as a marker for CTSCs in patients with colorectal cancer. This study demonstrated the prognostic significance of a combined carcinoembryonic antigen
(CEA)/cytokeratin (CK)/CD133 pattern but not for CD133 alone in patients with Dukes' B and C colorectal carcinoma (Iinuma et al., 2011). A marker "profile" that combines markers predicting poor clinical outcomes, resistance to chemotherapy, or susceptibility to apoptosis evasion (CD26, epidermal growth factor receptor, myeloid leukemia cell differentiation protein Mcl-1, and Ki-67) with the stem cell marker CD133 is currently under investigation. This approach is expected to result in improved ability to detect and target genuinely metastatic CTSC phenotypes (Faltas et al., 2011).

\section{APPROACHES FOR THERAPEUTIC TARGETING OF CIRCULATING TUMOR CELLS AND CIRCULATING TUMOR STEM CELLS}

Improved understanding of metastatic biology has laid the biological foundations for therapeutic targeting of CTCs and CTSCs. Table 1 summarizes important concepts in metastatic biology and their implications for therapeutic targeting. In vivo therapeutic targeting of CTCs and CTSCs to interrupt the transport phase of the metastatic cascade and subsequent development of metastasis is in itself, a novel concept (Hughes and King, 2012) and variety of targeting approaches are currently under development.

\section{Table 1 | Emerging concepts in metastatic biology and their implications for therapeutic targeting.}

\begin{tabular}{|c|c|c|}
\hline Biological concept & Implications for therapeutic targeting & Reference \\
\hline $\begin{array}{l}\text { - Metastasis progresses in a sequence of events. } \\
\text { - Each step in the metastatic cascade is considered } \\
\text { rate-limiting. }\end{array}$ & $\begin{array}{l}\text { Interrupting the hematogenous transport step can potentially } \\
\text { interrupt the entire cascade. }\end{array}$ & $\begin{array}{l}\text { Chambers et al. (1995), } \\
\text { Morris et al. (1997) }\end{array}$ \\
\hline - The parallel-progression model of metastatic tumors. & $\begin{array}{l}\text { Early targeting of CTC can eliminate early development of } \\
\text { micro-metastases. Anti-metastatic therapies may need to be } \\
\text { eliminated in conjunction with therapy for primary tumors. }\end{array}$ & Klein (2009) \\
\hline - Self-seeding of primary tumor. & $\begin{array}{l}\text { Therapeutic targeting of CTC even after the development of } \\
\text { secondary metastases may still alter the natural history } \\
\text { of the disease. }\end{array}$ & $\begin{array}{l}\text { Kim etal. (2009), } \\
\text { Comen et al. (2011) }\end{array}$ \\
\hline $\begin{array}{l}\text { - Hematogenous transport represents the final } \\
\text { common pathway of metastatic spread. }\end{array}$ & $\begin{array}{l}\text { Hematogenous transport is a rational therapeutic target to } \\
\text { prevent the development of metastases. }\end{array}$ & Sleeman et al. (2011) \\
\hline $\begin{array}{l}\text { - Hematogenous spread is an early event in the } \\
\text { metastatic cascade. }\end{array}$ & $\begin{array}{l}\text { The hematogenous transport phase provides a unique } \\
\text { therapeutic window for targeting an "up-stream" event in the } \\
\text { metastatic cascade. }\end{array}$ & Faltas (2010) \\
\hline $\begin{array}{l}\text { - Circulating tumor cells (CTCs) predict survival of } \\
\text { patients with metastatic cancers. }\end{array}$ & $\begin{array}{l}\text { Targeting circulating tumor cells could potentially prolong } \\
\text { survival of cancer patients. }\end{array}$ & $\begin{array}{l}\text { Cristofanilli etal. (2004), } \\
\text { Miller et al. (2010) }\end{array}$ \\
\hline $\begin{array}{l}\text { - Circulating tumor stem cells (CTSCs) capacity for } \\
\text { self-renewal, re-differentiation, dormancy, active DNA } \\
\text { repair, drug resistance, and resistance to apoptosis. }\end{array}$ & $\begin{array}{l}\text { CTSCs are considered high-value targets for anti-metastatic } \\
\text { therapies. Better profiling of CTSCs could have profound } \\
\text { implications for therapeutic targeting. }\end{array}$ & Faltas et al. (2011) \\
\hline $\begin{array}{l}\text { - CTCs can undergo a transformative process called } \\
\text { epithelial-mesenchymal transition (EMT) that leads }\end{array}$ & $\begin{array}{l}\text { Cell signaling pathways affecting regulation of EMT are } \\
\text { attractive therapeutic targets for anti-metastatic therapies. }\end{array}$ & Aalaoui-Jamali et al. (2011) \\
\hline $\begin{array}{l}\text { to the acquisition of enhanced migratory and } \\
\text { invasive properties. }\end{array}$ & $\begin{array}{l}\text { On the other hand EMT can alter surface marker expression } \\
\text { leading to decreased efficacy of anti-metastatic therapies } \\
\text { targeting specific epithelial surface markers. }\end{array}$ & \\
\hline
\end{tabular}


PHARMACOLOGICAL AND IMMUNOLOGICAL TARGETING APPROACHES Pharmacological targeting depends primarily on the identification of suitable molecular targets which are over-expressed and critically important for CTCs' survival and function. CTSC subsets are high-value targets because of their aggressive invasive and proliferative characteristics and their resistance to traditional chemotherapeutic agents. Current therapeutic approaches focusing on targeting CSCs in primary tumors could be extended to target CTSCs that express the same therapeutic targets.

Targeting surface receptors expressed preferentially in CSCs is one promising approach that could potentially be extended to CTSCs. In a study of human breast cancer xenografts in Swiss nude mice, an antibody targeting CD44, a surface marker expressed on CSCs, was found to reduce tumor growth and to prevent relapse after chemotherapy (Marangoni et al., 2009). In another study, targeting CD44 with short interfering RNA (siRNA) resulted in suppression of human colon cancer xenografts (Subramaniam et al., 2007). Investigators have also developed a fusion protein (dCD133KDEL) targeting CD 133, another CSC marker expressed by several human cancer cells (LaBarge and Bissell, 2008; Wang et al., 2008; Faltas et al., 2011). The fusion protein resulted in significant tumor reduction in a xenotransplant model of CD133+ human head and neck cancer (Waldron et al., 2011).

Epithelial cell adhesion molecule (EpCAM) is another interesting therapeutic target (Armstrong and Eck, 2003). It is frequently expressed on CSCs from breast, colon, pancreas, and prostate tumors (Gires et al., 2009). EpCAM is also expressed on CTCs but not on blood cells. This preferential expression is the basis of many diagnostic assays used to isolate CTCs from the bloodstream (Liljefors et al., 2005; Yu et al., 2011). Clinical trials using anti-EpCAM monoclonal antibodies slowed progression and prolonged survival in patient with metastatic colorectal carcinoma (Liljefors et al., 2005).

As mentioned earlier; CTSCs can arise through the EMT process (Bonnomet et al., 2010). Hence, signaling pathways affecting the regulation of EMT are attractive therapeutic targets for antimetastatic therapies. Inhibitors of PAR6A, Notch1, Hedgehog, Wnt, integrins, polycomb repressive complex 1 (PRC1) protein, Bmi-1, claudin, tyrosine kinase, and Rho GTPases have been reported to block EMT and a few select agents are undergoing early clinical trials (Aalaoui-Jamali et al., 2011).

In the future, a better understanding and profiling of shared characteristics between CTCs, CTSCs, and CSCs in different tumor subtypes will allow extension of the CSC targeting approaches mentioned above toward the design of specific anti-CTSC therapies. More clinical trials need to be designed to assess the impact of targeted pharmacological approaches against CTCs and CTSCs that aim to reduce metastases. These trials should include biomarker endpoints such CTC and CTSC counts and characteristics as well as survival endpoints to assess the full clinical impact of hindering metastasis development and to guide further drug development.

\section{IN VIVO DEVICE-BASED TARGETING APPROACHES}

Devices designed to target CTCs and CTSCs in vivo can be either intra-corporeal or extra-corporeal. Intra-corporeal devices such as indwelling vascular devices (Faltas, 2010) and shunts
(Wojciechowski et al., 2008) have the potential to continuously screen the entire circulation for CTC and CTSCs. This could theoretically improve the absolute magnitude of capture and ultimately result in greater improvements in clinical outcomes. On the other hand, intra-corporeal devices are susceptible to infection, clotting, and other physiological and anatomical design limitations (Faltas, 2010). Alternatively, extra-corporeal devices could isolate and destroy cancerous cells before returning the purged blood to the systemic circulation. Such design avoids many of the limitations inherent to intra-corporeal devices achieving higher capture efficiency per pass. However, the potential increase in capture efficiency per pass may be offset by limited contact time between the device and the circulation because extra-corporeal devices can only be used intermittently leaving periods of unopposed CTC dissemination. Some of these devices could also function as "theranostic" devices that simultaneously monitor CTC counts and analyze their biological characteristics while purging the circulation from CTCs. The resulting data could have prognostic as well as, predictive implications.

Circulating tumor cells and CTSCs can be isolated by targeting cell adhesion molecules expressed on their surfaces. One approach utilizes a vascular shunt functionalized with E-selectin to induce CTCs to adhere to and roll over the shunt's internal surface functionalized with immobilized TNF-related apoptosisinducing ligand (TRAIL) molecules. This device resulted in apoptosis of 30\% of CTCs after $1 \mathrm{~h}$ of rolling exposure (Rana et al., 2009). In another study, a P-selectin based vascular shunt functionalized with nanoscale liposomes containing siRNA was successful in delivering siRNA into leukemic cells inducing inhibition of target gene expression (Huang and King, 2009). The same approach can be extended to deliver siRNA into CTCs (Hughes and King, 2012).

Immuno-magnetic separation is another promising strategy that utilizes magnetic nano or micro-particles tagged with antibodies targeting surface receptors expressed by CTC/CTSC populations of interest followed by application of a magnetic field to capture cell-magnetic particle complexes. This principle is utilized by the CellSearch ${ }^{\circledR}$ diagnostic device approved for ex vivo separation of EpCAM+ CTCs in peripheral blood samples. The same concept is currently under development for in vivo applications. In one study aiming to prevent direct metastatic spread of ovarian cancer inside the peritoneal cavity, researchers used super-paramagnetic nanoparticles conjugated to a peptide targeting the EphA2 receptor expressed by ovarian cancer cells. The application of an external magnetic field resulted in cell capture from a flow stream in vitro and from the peritoneal cavity of experimental mice in vivo (Scarberry et al., 2008, 2010). In another study mice receiving this treatment were found to have tumor progression 10.77-times slower than that in the control group (Scarberry et al., 2011). The investigators envision the extension of this concept to produce a "dialysis-like" treatment that removes malignant cells from the body. This could potentially result in improved long-term survival of ovarian cancer patients (Scarberry et al., 2008).

On a parallel front, an extra-corporeal microfluidic immunomagnetic blood-cleansing device has been developed to treat sepsis. The device uses magnetic micro-beads coated with polyclonal 
antibodies to bind Candida albicans in the blood. Bead-fungi complexes were then subjected to a continuous magnetic gradient that enabled uninterrupted high throughput separation of fungi from whole blood. A multiplexed version of the device achieved over $80 \%$ clearance of fungi from contaminated blood at a flow rate of $20 \mathrm{ml} / \mathrm{h}$ in a single pass. The same technology can also be adapted to purge the blood from CTCs (Lee, 2009; Yung et al., 2009).

In vivo photo-acoustic flow cytometry is another emerging CTC separation technology. In one variant, melanin nanoparticles that naturally occur in melanoma CTCs were exposed to photons from a laser. The nanoparticles absorbed the photons generating heat energy that lysed CTCs while simultaneously expanding to produce acoustic waves detectable by an ultrasound transducer (Weight et al., 2006; Hughes and King, 2012). The same technique was applied to capture breast cancer CTCs using magnetic nanoparticles functionalized to target urokinase plasminogen activator, a receptor commonly expressed on breast CTCs. This was followed by photo-acoustic detection using gold-plated carbon nanotubes conjugated with folic acid as a second contrast agent (Galanzha et al., 2009a). In another study, photo-acoustic detection was used to target CD44+ CTSCs in a mouse model of human breast cancer (Galanzha et al., 2009b).

\section{CHALLENGES FACING THERAPEUTIC TARGETING OF CTC AND CTSCs}

Several challenges currently face CTC/CTSC therapeutic targeting efforts. CTCs are rare in the general circulation relative to other blood elements thus requiring ultra-efficient targeting. Pharmacological agents designed to target CTC/CTSCs need to achieve high selectivity while maintaining acceptable clinical toxicity, an important and sometimes an elusive goal. Furthermore, incomplete characterization and profiling of CTC populations hinders efforts to selectively target CTC subsets that are most likely to develop into metastases. CTSCs are emerging as a potentially high-value CTC subset but are still poorly defined and incompletely profiled to date (Faltas et al., 2011). Whereas a number of pharmacologic agents that target CSCs are under development (Aalaoui-Jamali et al., 2011), little is known regarding their specific effectiveness against CTSCs.

Device-based approaches face unique challenges of their own. While pre-clinical models have demonstrated proof of concept, many technical and biocompatibility issues need to be resolved before these devices reach clinical trials in humans. Such devices need to reach a certain threshold of capture efficiency while maintaining physiologic blood flow to prevent thrombosis or tumor seeding of the device (Faltas, 2010). Thromboembolic complications are to be anticipated with the use of such devices and while these complications can be prevented or managed with concomitant use of anticoagulants, this introduces significant bleeding risks that may decrease the overall clinical benefit. Bloodstream infection related to indwelling vascular devices is another serious clinical problem that could potentially complicate clinical

\section{REFERENCES}

Aalaoui-Jamali, M., Bijian, K., and Batist, G. (2011). Emerging drug discovery approaches for selective targeting of "precursor" metastatic breast cancer cells: highlights and perspectives. Am. J. Transl. Res. 3, 434-444.

adoption of such technology. Approaches utilizing functionalized surfaces face challenges related to bio-fouling by plasma proteins and blood elements that alter the biological activity of such functionalized surfaces (Hughes and King, 2012). Systems utilizing magnetic nanoparticles will require more robust data on the potential long-term toxicity of these particles when used in vivo (Scheinberg et al., 2010). Using animal-derived antibodies to isolate CTCs in humans could potentially induce hypersensitivity reactions and while humanized antibodies can be synthesized, they are generally more expensive.

\section{CONCLUSION}

Recent insights into the biology of the metastasis have improved our understanding of the metastatic cascade and potential therapeutic targets. These insights have laid the biological foundations for therapeutic targeting of CTCs, a promising area of research that aims to reduce cancer morbidity and mortality by preventing the development of metastases at a very early stage. Studies reveal a multi-step metastatic process that is complex, dynamic, and multi-directional. Metastatic cancer cells disseminate very early in the course of solid tumor growth forming metastases that grow in parallel with the primary tumor. The crucial role of CTCs and CTSCs in initiating and perpetuating metastases is increasingly being recognized. Despite challenges facing precise identification of CTSCs, they are likely to emerge as attractive therapeutic targets because of their stem cell like properties. Several pharmacological and immunological CSC targeting agents are being developed that may eventually prove effective against CTSCs sharing the same biological characteristics.

The hematogenous transport phase of the metastatic cascade provides critical access to CTCs and CTSCs that permits therapeutic targeting. Recent advances in the fields of nanotechnology and microfluidics have led to the development of several devices for in vivo targeting of CTC during transit in the circulation. Selectin-coated tubes that target cell adhesion molecules, immuno-magnetic separators, and in vivo photoacoustic flow cytometers are currently being developed for this purpose. While pre-clinical models are showing promising preliminary results, several technical and biocompatibility issues need to be resolved before these devices could reach clinical trials on humans.

Although still in its infancy, therapeutic targeting of CTCs and CTSCs offers an unprecedented opportunity to prevent the development of metastasis and potentially alter the natural history of cancer. More research is needed to expedite the translation of these promising technologies to clinical therapies. By rendering cancer a "local" disease, these approaches could lead to major reductions in metastasis-related morbidity and mortality.

\section{ACKNOWLEDGMENT}

The author would like to thank Paul L. Bernstein, MD for his critical review of the manuscript.

Armstrong, A., and Eck, S. L. (2003). EpCAM: a new therapeutic target for an old cancer antigen. Cancer Biol. Ther. 2, 320-326.
Bonnomet, A., Brysse, A., Tachsidis, A., Waltham, M., Thompson, E. W., Polette, M., and Gilles, C. (2010). Epithelial-to-mesenchymal 
transitions and circulating tumor cells. J. Mammary Gland Biol. Neoplasia 15, 261-273.

Chaffer, C. L., and Weinberg, R. A. (2011). A perspective on cancer cell metastasis. Science 331, 1559-1564.

Chambers, A. F., MacDonald, I. C., Schmidt, E. E., Koop, S., Morris, V. L., Khokha, R., and Groom, A. C. (1995). Steps in tumor metastasis: new concepts from intravital videomicroscopy. Cancer Metastasis Rev. 14, 279-301.

Chambers, A. F., MacDonald, I. C., Schmidt, E. E., Morris, V. L., and Groom, A. C. (2000). Clinical targets for anti-metastasis therapy. $A d v$. Cancer Res. 79, 91-121.

Comen, E., Norton, L., and Massague, J. (2011). Clinical implications of cancer self-seeding. Nat. Rev. Clin. Oncol. 8, 369-377.

Cristofanilli, M., Budd, G. T., Ellis, M. J., Stopeck, A., Matera, J., Miller, M. C., Reuben, J. M., Doyle, G. V., Allard, W. J., Terstappen, L. W., and Hayes, D. F. (2004). Circulating tumor cells, disease progression, and survival in metastatic breast cancer. N. Engl. J. Med. 351, 781-791.

Duffy, M. J., McGowan, P. M., and Gallagher, W. M. (2008). Cancer invasion and metastasis: changing views. J. Pathol. 214, 283-293.

Faltas, B. (2010). Targeting hematogenous spread of circulating tumor cells by a chemotactic drug-eluting IVC filter to prevent pulmonary and systemic metastasis. Med. Hypotheses 74, 668-669.

Faltas, B., Zeidan, A., Peters, K., Das, A., Joudeh, J., Navaraj, A., Dolloff, N. G., Harvey, H. A., Jiang, Y., Allen, J. E., Dicker, D. T., and El Deiry, W. S. (2011). Identifying circulating tumor stem cells that matter: the key to prognostication and therapeutic targeting. J. Clin. Oncol. 29, 2946-2947; author reply 2947-2948.

Galanzha, E. I., Shashkov, E. V., Kelly, T., Kim, J. W., Yang, L., and Zharov, V. P. (2009a). In vivo magnetic enrichment and multiplex photoacoustic detection of circulating tumour cells. Nat. Nanotechnol. 4, 855-860.

Galanzha, E. I., Kim, J. W., and Zharov, V. P. (2009b). Nanotechnology-based molecular photoacoustic and photothermal flow cytometry platform for in-vivo detection and killing of circulating cancer stem cells. J. Biophotonics 2, 725-735.

Gires, O., Klein, C. A., and Baeuerle, P.A. (2009). On the abundance of EpCAM on cancer stem cells. Nat. Rev. Cancer 9, 143; author reply 143 .

Huang, Z., and King, M. R. (2009). An immobilized nanoparticlebased platform for efficient gene knockdown of targeted cells in the circulation. Gene Ther. 16, 1271-1282.

Hughes, A. D., and King, M. R. (2012). Nanobiotechnology for the capture and manipulation of circulating tumor cells. Wiley Interdiscip. Rev. Nanomed. Nanobiotechnol. 4, 291-309.

Iinuma, H., Watanabe, T., Mimori, K., Adachi, M., Hayashi, N., Tamura, J., Matsuda, K., Fukushima, R., Okinaga, K., Sasako, M., and Mori, M. (2011). Clinical significance of circulating tumor cells, including cancer stem-like cells, in peripheral blood for recurrence and prognosis in patients with dukes' stage $B$ and $C$ colorectal cancer. J. Clin. Oncol. 29, 1547-1555.

Kim, M. Y., Oskarsson, T., Acharyya, S., Nguyen, D. X., Zhang, X. H., Norton, L., and Massague, J. (2009). Tumor self-seeding by circulating cancer cells. Cell 139, 1315-1326.

Klein, C. A. (2009). Parallel progression of primary tumours and metastases. Nat. Rev. Cancer 9, 302-312.

Kruck, S., Gakis, G., and Stenzl, A. (2011). Disseminated and circulating tumor cells for monitoring chemotherapy in urological tumors. Anticancer Res. 31, 2053-2057.

LaBarge, M. A., and Bissell, M. J. (2008). Is CD133 a marker of metastatic colon cancer stem cells? J. Clin. Invest. 118, 2021-2024.

Lee, A. A. (2009). Cutting edge: microfluidic-micromagnetic blood cleansing device. Lab Chip 9, 1167.

Liljefors, M., Nilsson, B., Fagerberg, J., Ragnhammar, P., Mellstedt, H., and Frodin, J. E. (2005). Clinical effects of a chimeric anti-EpCAM monoclonal antibody in combination with granulocyte-macrophage colony-stimulating factor in patients with metastatic colorectal carcinoma. Int. J. Oncol. 26, 1581-1589.

MacDonald, I. C., Groom, A. C., and Chambers, A. F. (2002). Cancer spread and micrometastasis development: quantitative approaches for in vivo models. Bioessays 24, 885-893.

Marangoni, E., Lecomte, N., Durand, L., de Pinieux, G., Decaudin, D., Chomienne, C., Smadja-Joffe, F., and Poupon, M. F. (2009). CD44 targeting reduces tumour growth and prevents post-chemotherapy relapse of human breast cancers xenografts. Br. J. Cancer 100, 918-922.

Miller, M. C., Doyle, G. V., and Terstappen, L. W. (2010). Significance of circulating tumor cells detected by the CellSearch system in patients with metastatic breast colorectal and prostate cancer. J. Oncol. 2010, 617421.
Mina, L. A., and Sledge, G. W. Jr. (2011). Rethinking the metastatic cascade as a therapeutic target. Nat. Rev. Clin. Oncol. 8, 325-332.

Monteiro, J., and Fodde, R. (2010). Cancer stemness and metastasis: therapeutic consequences and perspectives. Eur. J. Cancer 46, 1198-1203.

Morris, V. L., Schmidt, E. E., MacDonald, I. C., Groom, A. C., and Chambers, A. F. (1997). Sequential steps in hematogenous metastasis of cancer cells studied by in vivo videomicroscopy. Invasion Metastasis 17, 281-296.

Nichols, A. C., Lowes, L. E., Szeto, C. C. Basmaji, J., Dhaliwal, S., Chapeskie, C., Todorovic, B., Read, N., Venkatesan, V., Hammond, A., Palma, D. A., Winquist, E., Ernst, S., Fung, K., Franklin, J. H., Yoo, J., Koropatnick, J., Mymryk, J. S., Barrett, J. W., and Allan, A. L. (2011). Detection of circulating tumor cells in advanced head and neck cancer using the cellsearch system. Head Neck. doi: 10.1002/hed.21941 [Epub ahead of print].

O'Flaherty, J. D., Gray, S., O’Leary, J. J., Blackhall, F. H., and O’Byrne, K. J. (2011). Circulating tumour cells, their role in metastasis and their clinical utility in lung cancer. Lung Cancer 76, 19-25.

Rana, K., Liesveld, J. L., and King, M. R. (2009). Delivery of apoptotic signal to rolling cancer cells: a novel biomimetic technique using immobilized TRAIL and E-selectin. Biotechnol. Bioeng. 102, 1692-1702.

Scarberry, K. E., Dickerson, E. B., McDonald, J. F., and Zhang, Z. J. (2008). Magnetic nanoparticlepeptide conjugates for in vitro and in vivo targeting and extraction of cancer cells. J. Am. Chem. Soc. 130, 10258-10262.

Scarberry, K. E., Dickerson, E. B., Zhang, Z. J., Benigno, B. B., and McDonald, J. F. (2010). Selective removal of ovarian cancer cells from human ascites fluid using magnetic nanoparticles. Nanomed. Nanotechnol. Biol. Med. 6, 399-408.

Scarberry, K. E., Mezencev, R., and McDonald, J. F. (2011). Targeted removal of migratory tumor cells by functionalized magnetic nanoparticles impedes metastasis and tumor progression. Nanomedicine (Lond.) 6, 69-78.

Scheinberg, D. A., Villa, C. H., Escorcia, F. E., and McDevitt, M. R. (2010). Conscripts of the infinite armada: systemic cancer therapy using nanomaterials. Nat. Rev. Clin. Oncol. 7, 266-276.

Sleeman, J. P., Nazarenko, I., and Thiele, W. (2011). Do all roads lead to Rome? routes to metastasis development. Int. J. Cancer 128, 2511-2526.

Subramaniam, V., Vincent, I. R., Gilakjan, M., and Jothy, S. (2007). Suppression of human colon cancer tumors in nude mice by siRNA CD44 gene therapy. Exp. Mol. Pathol. 83, 332-340.

Swaby, R. F., and Cristofanilli, M. (2011). Circulating tumor cells in breast cancer: a tool whose time has come of age. BMC Med. 9, 43. doi: 10.1186/1741-7015-9-43

Takeuchi, H., and Kitagawa, Y. (2010). Circulating tumor cells in gastrointestinal cancer. J. Hepatobiliary Pancreat. Sci. 17, 577-582.

Valastyan, S., and Weinberg, R. A. (2011). Tumor metastasis: molecular insights and evolving paradigms. Cell 147, 275-292.

Waldron, N. N., Kaufman, D. S., Oh, S., Inde, Z., Hexum, M. K., Ohlfest, J. R., and Vallera, D. A. (2011). Targeting tumor-initiating cancer cells with dCD133KDEL shows impressive tumor reductions in a xenotransplant model of human head and neck cancer. Mol. Cancer Ther. 10, 1829-1838.

Wang, J., Sakariassen, P. O., Tsinkalovsky, O., Immervoll, H., Boe, S. O., Svendsen, A., Prestegarden, L., Røsland, G., Thorsen, F., Stuhr, L., Molven, A., Bjerkvig, R., and Enger, P. Ø. (2008). CD133 negative glioma cells form tumors in nude rats and give rise to CD133 positive cells. Int. J. Cancer 122 , 761-768.

Weight, R. M., Viator, J. A., Dale, P. S., Caldwell, C. W., and Lisle, A. E. (2006). Photoacoustic detection of metastatic melanoma cells in the human circulatory system. Opt. Lett. 31, 2998-3000.

Wittekind, C., and Neid, M. (2005). Cancer invasion and metastasis. Oncology 69(Suppl. 1), 14-16.

Wojciechowski, J. C., Narasipura, S. D., Charles, N., Mickelsen, D., Rana, K., Blair, M. L., and King, M. R. (2008). Capture and enrichment of CD34-positive haematopoietic stem and progenitor cells from blood circulation using P-selectin in an implantable device. Br. J. Haematol. 140, 673-681.

Wong, C. W., Lee, A., Shientag, L., Yu, J., Dong, Y., Kao, G., Al-Mehdi, A. B., Bernhard, E. J., and Muschel, R. J. (2001). Apoptosis: an early event in metastatic inefficiency. Cancer Res. 61, 333-338.

Yu, M., Stott, S., Toner, M., Maheswaran, S., and Haber, D. A. (2011). Circulating tumor cells: approaches to isolation and characterization. $J$. Cell Biol. 192, 373-382. 
Yung, C. W., Fiering, J., Mueller, A. J., and Ingber, D. E. (2009). Micromagnetic-microfluidic blood cleansing device. Lab Chip 9, 1171-1177.

Conflict of Interest Statement: The author declares that the research was conducted in the absence of any commercial or financial relationships that could be construed as a potential conflict of interest.

Received: 01 June 2012; paper pending published: 11 June 2012; accepted: 12 June 2012; published online: 03 July 2012.
Citation: Faltas B (2012) Cornering metastases: therapeutic targeting of circulating tumor cells and stem cells. Front. Oncol. 2:68. doi: 10.3389/fonc.2012. 00068

This article was submitted to Frontiers in Cancer Molecular Targets and Therapeutics, a specialty of Frontiers in Oncology.
Copyright (C) 2012 Faltas. This is an open-access article distributed under the terms of the Creative Commons Attribution Non Commercial License, which permits non-commercial use, distribution, and reproduction in other forums, provided the original authors and source are credited. 